Role of defects and their complexes on the dependence of photoconductivity on dark resistivity of single $\mathrm{ZnO}$ microwires

M. Villafuerte, D. J. Zamora, G. Bridoux, J. M. Ferreyra, M. Meyer, and S. P. Heluani

Citation: J. Appl. Phys. 121, 064501 (2017); doi: 10.1063/1.4975197

View online: http://dx.doi.org/10.1063/1.4975197

View Table of Contents: http://aip.scitation.org/toc/jap/121/6

Published by the American Institute of Physics

A|P $\left.\right|_{\text {Applied Physics }} ^{\text {Journal of }}$

INTRODUCING INVITED PERSPECTIVES

Ultrafast magnetism and $\mathrm{THz}$ spintronics

Authors: Jakob Walowski and Markus Münzenberg 


\title{
Role of defects and their complexes on the dependence of photoconductivity on dark resistivity of single $\mathrm{ZnO}$ microwires
}

\author{
M. Villafuerte, ${ }^{1, a)}$ D. J. Zamora, ${ }^{2}$ G. Bridoux, ${ }^{1}$ J. M. Ferreyra, ${ }^{2}$ M. Meyer, ${ }^{3}$ and S. P. Heluani ${ }^{2}$ \\ ${ }^{1}$ Laboratorio de Física del Sólido, Dpto. de Física, FCEyT, Universidad Nacional de Tucumán and CONICET, \\ 4000 S.M. de Tucumán, Argentina \\ ${ }^{2}$ Laboratorio de Física del Sólido, Dpto. de Física, FCEyT, Universidad Nacional de Tucumán, \\ 4000 S.M. de Tucumán, Argentina \\ ${ }^{3}$ Instituto de Física, Dpto. de Física, Fac. de Ciencias Exactas, Universidad Nacional de La Plata, \\ 1900 La Plata, Argentina
}

(Received 31 October 2016; accepted 18 January 2017; published online 9 February 2017)

\begin{abstract}
We have studied the correlation between the photoconductivity and the dark resistivity of single $\mathrm{ZnO}$ microwires. We found that as-grown microwires with higher dark resistivities have higher photoconductivities. However, when the microwires are thermal treated in vacuum, this correlation is inverted. We have also analyzed the behavior of photoconductivity on protonated as-grown samples. We discuss the origin of these behaviors in terms of the interplay of oxygen and zinc vacancies and their complexes acting as recombination or trapping centers. Published by AIP Publishing.
\end{abstract}

[http://dx.doi.org/10.1063/1.4975197]

\section{INTRODUCTION}

Ultraviolet (UV) photodetectors based on wide-bandgap oxides have been intensively investigated due to their intrinsic visible-blindness, high thermal, and chemical stability. ${ }^{1}$ In particular, $\mathrm{ZnO}$ has drawn great interest due to its low cost, tunable bandgap, and ability to be operable in harsh environments. ${ }^{2}$ Furthermore, in order to enhance the photosensitivity and speed of the devices based on $\mathrm{ZnO}$, several attempts were made, for instance: surface-to-volume ratio, ${ }^{3,4}$ decoration with semiconductors ${ }^{5}$ or metals ${ }^{6-8}$ with subsequent diffusion, ${ }^{9}$ doping with metals or no-metals, ${ }^{10-13}$ and formation of cap layers. ${ }^{14,15} \mathrm{~A} \mathrm{ZnO} \mathrm{UV}$ photodetector has been fabricated by ultraviolet nanoimprint lithography with hydrothermal synthesis in which it was produced as Schottky barriers in order to get an ultrafast response. ${ }^{16}$ The recovery time of a thin film transistor based UV photodetector was improved by the suppression of the persistent photoconductivity through the oxygen plasma treatment of the $\mathrm{ZnO}$ channel surface. ${ }^{17}$ Also, an enhancement of the photoresponse can be obtained by simultaneously applying a gate bias and UV illumination in an Electric Double Layer Field Effect Transistor configuration. ${ }^{18}$ These results make it clear that a profound knowledge of the physics of defects in $\mathrm{ZnO}$ is necessary in order to improve the efficiency of the photodetectors. In this way, it is worth to study the influence of the intrinsic defects on the photoresponse of $\mathrm{ZnO}$.

In this work, we study the photoresponse of single $\mathrm{ZnO}$ microwires (MWs) and its correlation with the resistivity in the dark $\left(\rho_{d}\right)$. Our hypothesis is that the magnitude of the dark resistivity is mainly established by the amount of intrinsic point defects in a particular MW. Furthermore, in order to study this correlation under a change in the imperfection content, the samples were annealed in vacuum or doped with hydrogen by diffusion at high pressures and temperatures. We

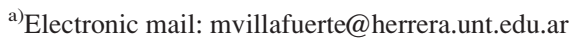

prove here that, in as-grown samples, as the $\rho_{d}$ increases, the photoresponse is greater, and in annealed samples in vacuum, that correlation is dramatically inverted, while the hydrogenated samples did show a more complicated behavior. Moreover, we develop a simple model based on our experimental results to give the explanation of these behaviors.

\section{EXPERIMENTAL DETAILS}

For this study, MWs of $\mathrm{ZnO}$ were prepared by the carbothermal process consistent in the thermal decomposition of high melting point $\mathrm{ZnO}\left(1975^{\circ} \mathrm{C}\right)$ into low melting point $\mathrm{Zn}$ or $\mathrm{Zn}$ suboxides $\left(419^{\circ} \mathrm{C}\right)$. For carbothermal evaporation, ${ }^{19}$ a pressed $\mathrm{ZnO} /$ graphite target (mass ratio of $1: 1$ ) was placed on a ceramic holder inside a quartz tube. When the temperature of a programmable tubular furnace had reached $1150^{\circ} \mathrm{C}$, the quartz tube was inserted into it. Both outlets of the quartz tube were kept open to the ambient air, and no carrier gas was applied. The reaction time was of $45 \mathrm{~min}$. The wires grow directly on the targets with diameters from 10 to $40 \mu \mathrm{m}$ and lengths of some millimeters (see Fig. 1(a)). The obtained microwires were hexagonal shaped with flat surfaces, as can be seen in Fig. 1(b). Part of the samples was thermal treated (TT) at $700^{\circ} \mathrm{C}$ during 2 or $5 \mathrm{~h}$ at $10^{-3}$ Torr in order to enhance the intrinsic oxygen vacancy content. Other part of the as-grown samples was protonated by the diffusion of hydrogen. Two different processes were used: 30 bar of $\mathrm{H}_{2}$ at $300^{\circ} \mathrm{C}$ for $100 \mathrm{~min}$ or 40 bar of $\mathrm{H}_{2}$ at $450{ }^{\circ} \mathrm{C}$ for $12 \mathrm{~h}$. The Raman spectra of the samples were recorded at room temperature by employing a diode-pump, a solid state $532 \mathrm{~nm}$ laser (spot of $\approx 2 \mu \mathrm{m}$ ), a Thermo Scientific DXR Raman Microscope equipped with a CCD detector. XAS measurements were taken at room temperature in the transmission mode at the Zn K-edge, at the XAFS beam-line of LNLS (Campinas, Brazil). Ohmic contacts with linear and symmetric I-V curves were achieved by using In contacts on the MWs (see Fig. 1(c)). Resistivity measurements were 


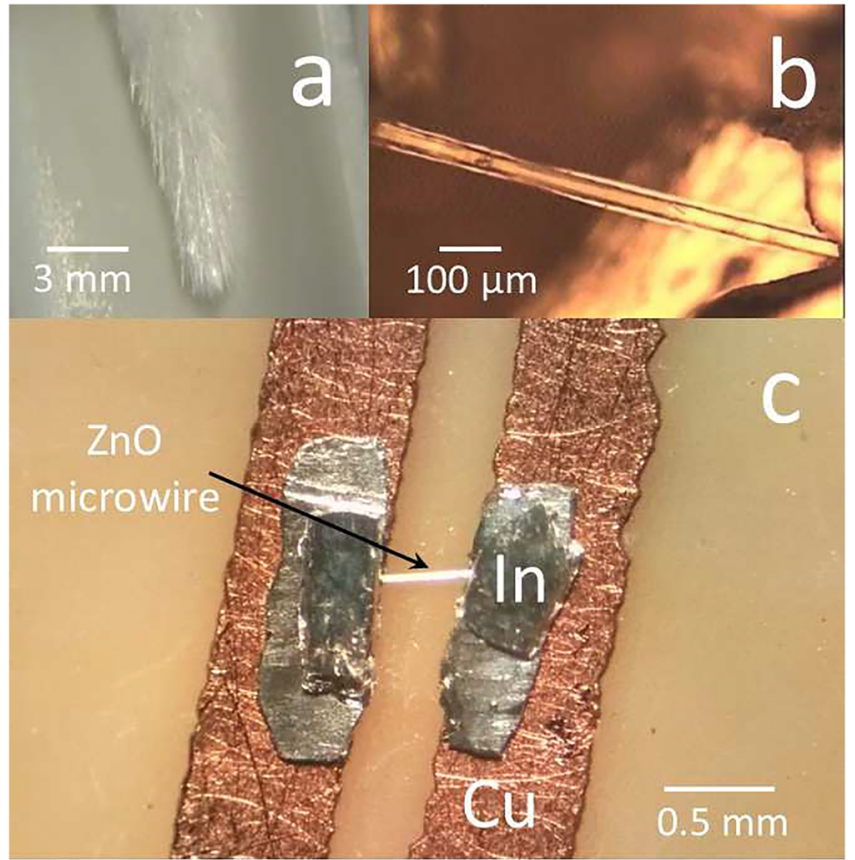

FIG. 1. (a) Image of a bunch of as-fabricated microwires obtained by carbothermal evaporation. (b) Image of a single microwire showing the hexagonal faceted surface. (c) Photography of the setup used to measure the phototransport properties. The microwire is cold soldered to the $\mathrm{Cu}$ pads using $\mathrm{In}$.

performed in a voltage source-ammeter configuration. An excitation voltage of $5 \mathrm{~V}$ was used, and the electrical current was measured with a resolution of $0.5 \mathrm{nA}$. Measurements were carried out in a standard cryostat equipped with an optical window and a $1000 \mathrm{~W}$ Xe lamp plus a monochromator. The wavelength can be swept from 200 to $800 \mathrm{~nm}$ with an estimated flux density of $10 \mu \mathrm{W} / \mathrm{cm}^{2}$ in the UV range.

\section{RESULTS AND DISCUSSION}

Fig. 2 shows the Raman spectra of representative MWs: as grown, thermal treated in vacuum (samples TT $2 \mathrm{~h}$ and TT $5 \mathrm{~h}, 2 \mathrm{~h}$ and $5 \mathrm{~h}$ at $700^{\circ} \mathrm{C}$, respectively) and protonated by diffusion (sample $\mathrm{H}$ ). All peaks correspond to the Raman

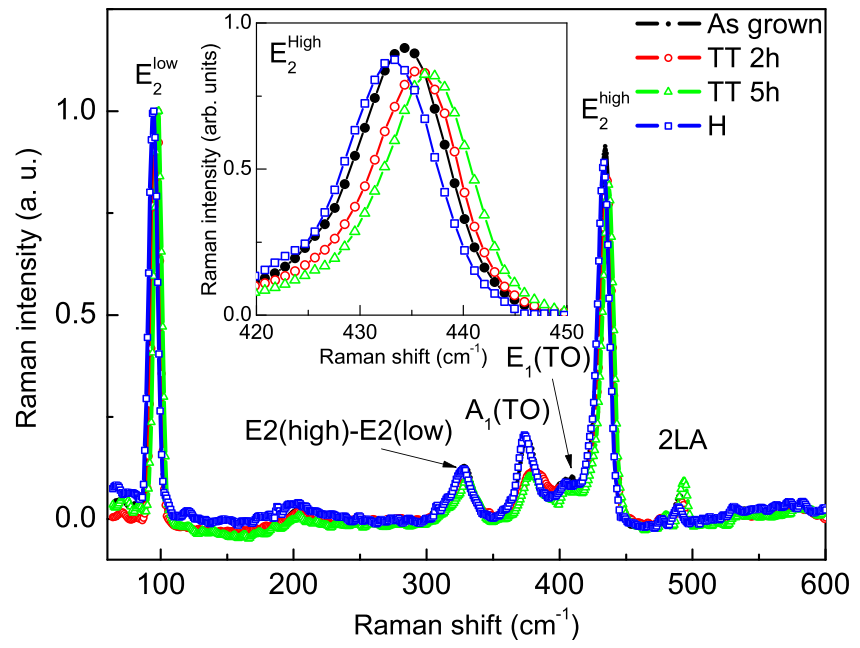

FIG. 2. Raman spectra of single $\mathrm{ZnO}$ microwires. Inset: Zoom of the E2(high) peak. spectrum of wurtzite $\mathrm{ZnO} .^{20} \mathrm{We}$ measured the spectra in several MWs, and we repeated each spectrum in order to check the repeatability of our micro-Raman setup. The spectra were normalized using their respective E2(low) peak intensity. The inset shows a zoom of the E2(high) peaks where we can see that the relative intensity for TT samples is lower than the intensity of the same peak corresponding to the as-grown sample. The low-wavenumber E2(low) mode predominantly involves the vibration of the heavy $\mathrm{Zn}$ sublattice, while the high-wavenumber E2(high) mode is mainly associated with the vibration of the lighter $\mathrm{O}$ sublattice. Then, the diminution of the intensity of E2(high) suggests a lesser oxygen content in TT samples, as expected. Moreover, the spectra of vacuum treated samples shift to a higher Raman shift in relation to as-grown samples. The shift to higher frequencies of the E2 (high) mode indicates a stress effect of the vacancies in the structure of $\mathrm{ZnO}$. The A1(TO) mode at $380 \mathrm{~cm}^{-1}$ is related to oscillation modes parallel to the $\mathrm{c}$ axis and mainly to the lighter oxygen sublattice. We observe that the diminution of intensity goes in the same direction as E2(high) for TT samples. The spectra for $\mathrm{H}$ samples show intensities similar to as-grown samples with a slight tendency to shift to lower frequencies, indicating that the strain is opposite as compared with TT samples.

EXAFS measurement provides detailed information on the local structure around $\mathrm{Zn}$ atoms in $\mathrm{ZnO}$ MWs. Fig. 3 shows the Fourier transform (FT) of oscillations extracted from the Zn k-edge, using an Athena program, corresponding to AG and TT MWs. From this figure, it can be observed, out of the error, the reduction of the EXAFS amplitude of the first ( $\mathrm{Zn}-\mathrm{O}$ bond distance) and second shell ( $\mathrm{Zn}-\mathrm{Zn}$ bond distance) as the thermal treatment time increases (see inset in the same figure). This qualitative analysis suggests an increasing disorder of the atomic structure due to the existence of $\mathrm{O}$ and $\mathrm{Zn}$ vacancies.

MWs of the same geometrical aspect were selected to perform the photo-transport measurements: mean length between the contacts of $312 \mu \mathrm{m}$ (standard deviation, $\mathrm{SD}=100 \mu \mathrm{m}$ ) and

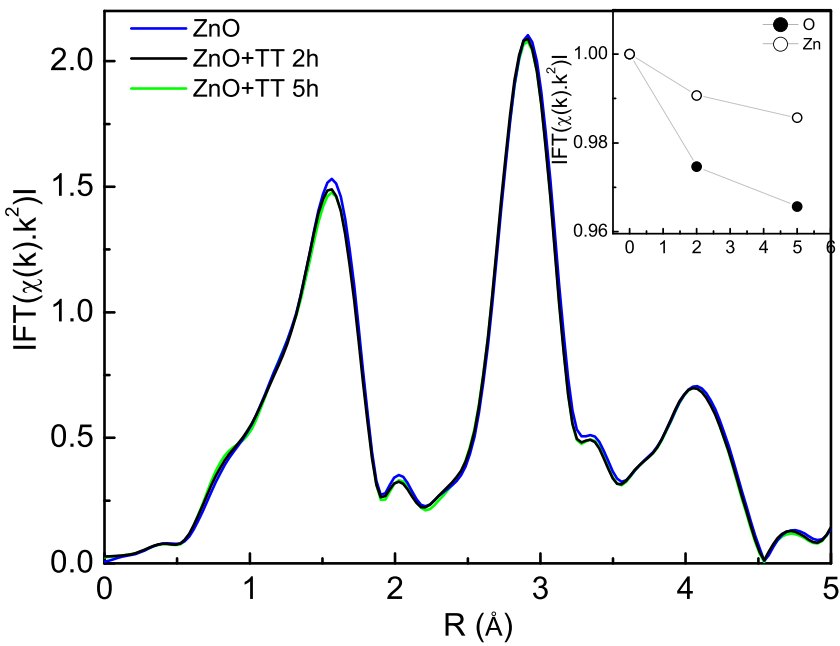

FIG. 3. Fourier transform modulus of $\chi(\mathrm{k}) \cdot \mathrm{k}^{2}$ EXAFS of as-grown (AG) and thermal treated in vacuum (TT) $\mathrm{ZnO}$ microwires. The inset shows the decrease of oxygen (filled symbols) and zinc (hollow symbols) $\chi(\mathrm{k}) \cdot \mathrm{k}^{2} \mathrm{max}-$ ima normalized to the $\mathrm{AG}$ value as a function of thermal treatment time. 
the mean diameter of $25 \mu \mathrm{m}(\mathrm{SD}=3 \mu \mathrm{m})$. Photoconductivity is defined as: $\left(\sigma_{\text {light }}-\sigma_{\text {dark }}\right) / \sigma_{\text {dark }}$, where $\sigma_{\text {light }}$ is the conductivity with illumination and $\sigma_{\text {dark }}$ is the conductivity in the dark. The photoconductivity growth curves of the MWs using a light of $\lambda=370 \mathrm{~nm}$ in vacuum $\left(\approx 10^{-3}\right.$ Torr $)$ at roomtemperature are shown in Fig. 4. Each measurement was made after maintaining the MWs in the dark for $24 \mathrm{~h}$. The curves show a great variation of responses to the UV, depending on the dark resistivity of the MWs. The dark resistivity was calculated as: $\rho_{\text {dark }}=1 / \sigma_{\text {dark }}=\left(\mathrm{V} / I_{\text {dark }}\right)$. A $/ 1$, where $I_{\text {dark }}$ is the current in the dark, and A and 1 are the area of cross section and length between the contacts of the MWs, respectively. For as grown MWs, the PC increases as the dark resistivity increases. However, in TT MWs, the trend is inverted: the PC increases for decreasing dark resistivity.

The best fitting of the photoconductivity growth curves was achieved by double exponential functions

$$
\mathrm{PC}=\mathrm{y}_{0}+\mathrm{A}_{1} \exp \left(\mathrm{t} / \tau_{1}\right)+\mathrm{A}_{2} \exp \left(\mathrm{t} / \tau_{2}\right)
$$

where $\tau_{1}$ and $\tau_{2}$ are the fast and slow time constants, respectively (see Fig. 5), and $\mathrm{y}_{0}, \mathrm{~A}_{1}$, and $\mathrm{A}_{2}$ are fitting constants. Moore and Thompson ${ }^{21}$ developed a phenomenological model that explains these responses in terms of two processes for the charge transfer: bulk-to-surface transfer of carriers through the potential energy barrier, due to surface band bending; and thermionic surface-to-bulk transfer of charge carriers through a fixed potential energy barrier. In the dark, the surface of the MWs has adsorbed $O_{2}$ that captures electrons increasing the band bending. The magnitude of the depletion width will depend on the balance between donors and acceptors near the surface. When the sample is illuminated with UV light, bulk-to-surface transfer of holes dominates and, depending on the initial amount of band bending, can result in a significant initial growth in photocurrent, that is, a fast process characterized by the time constant $\tau_{1}$. As the band bending decreases with time, due to the desorption of $\mathrm{O}_{2}$, the rate of transfer bulk-to-surface

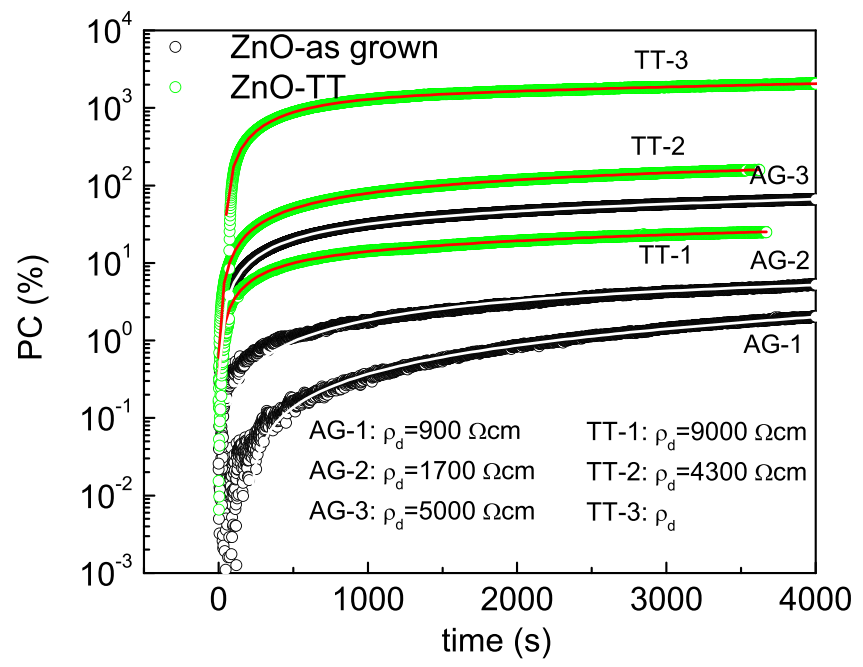

FIG. 4. Growth of photoconductivity of representative as-grown (AG) and thermal treated in vacuum (TT) $\mathrm{ZnO}$ microwires using $\lambda=370 \mathrm{~nm}$. The dark resistivities are indicated for each sample. The lines indicate the fitting data using a double exponential function.

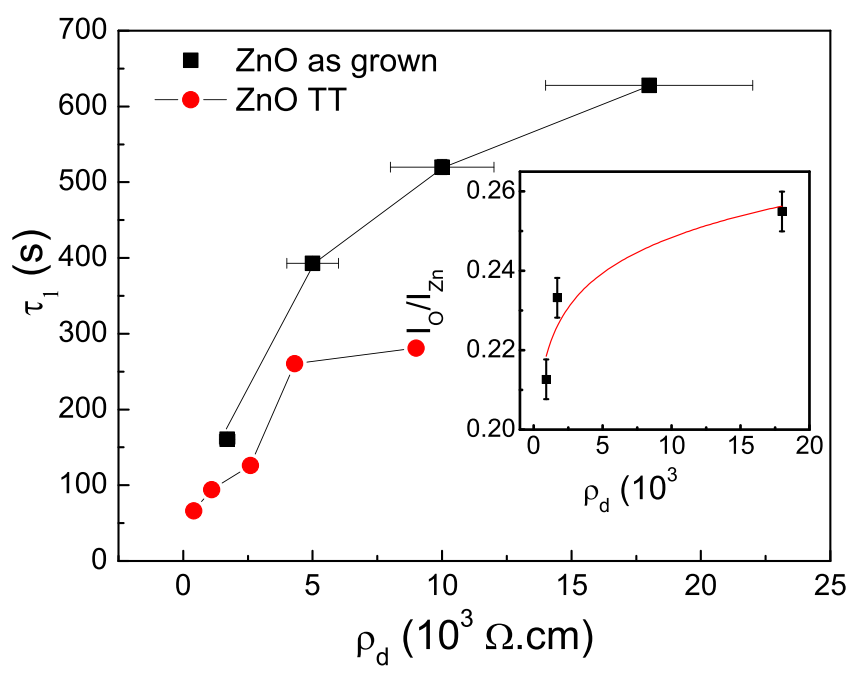

FIG. 5. Photoconductivity growth fast time constants $\left(\tau_{1}\right)$ of AG and TT samples, obtained by fitting with double exponentials. Inset: Ratio of intensities of O-Ka and Zn-La lines of Energy-Dispersive X-ray Spectroscopy data for as-grown samples. The line is a guide for the eye.

competes with the surface-to-bulk transfer. This slowing of photocurrent is typically ascribed as the slow process, characterized by the time constant $\tau_{2}{ }^{22}$ In order to compare the time response of different samples, one must be aware that this comparison will be valid only at the same ambient pressure and the surface-to-volume ratio. The increase in $\tau_{1}$ (Fig. 5) indicates that the bulk-to-surface transfer is slower at the beginning of illumination as $\rho_{d}$ increases. This can be interpreted in terms of lower band bending at the initial stage as $\rho_{d}$ increases. ${ }^{23}$ The presence of acceptors, such as zinc vacancies $V_{Z n},{ }^{24,25}$ must be a plausible explanation. Then, as the concentration of $V_{Z n}$ increases, thus partially compensating the intrinsic donor defects, the $\rho_{d}$ becomes greater. In order to check this hypothesis, we performed the energydispersive X-ray spectroscopy in two points of each microwire and plotted the mean ratio of the intensity of the O-Ka line over the intensity of the $\mathrm{Zn}-\mathrm{La}$ line vs. $\rho_{d}$ (see inset Fig. 5(a)). This ratio represents the change of concentration of oxygen in relation to the concentration of $\mathrm{Zn}$ in the samples. The plot shows that the concentration of $\mathrm{Zn}$ is diminishing in relation to $\mathrm{O}$ as $\rho_{d}$ increases, which can be interpreted as an increase of the concentration of $V_{Z n}$. In the case of the TT-ZnO samples, the tendency of the $\tau_{1}$ is the same as the as-grown samples, but with lower values, indicating the presence of a lower band bending at the beginning stage before illumination.

Fig. 6 shows the absolute value of $\mathrm{PC}$ as a function of the dark resistivity $\left(\rho_{d}\right)$ of single MWs for as grown and TT samples. The values of PC were taken from Fig. 4 at one hour of illumination. There were no essential changes in the main features of these curves for other times. The range of dark resistivities change one order of magnitude, which means that the concentration of majority carriers has the same variation, assuming that the mobility is nearly constant. As grown MWs show a saturation of PC around $100 \%$. However, in the case of TT MWs, the highest PC reaches $1000 \%$. The points of the lowest PC correspond to TT- $2 \mathrm{~h}$ samples and the rest to TT-5h samples. 


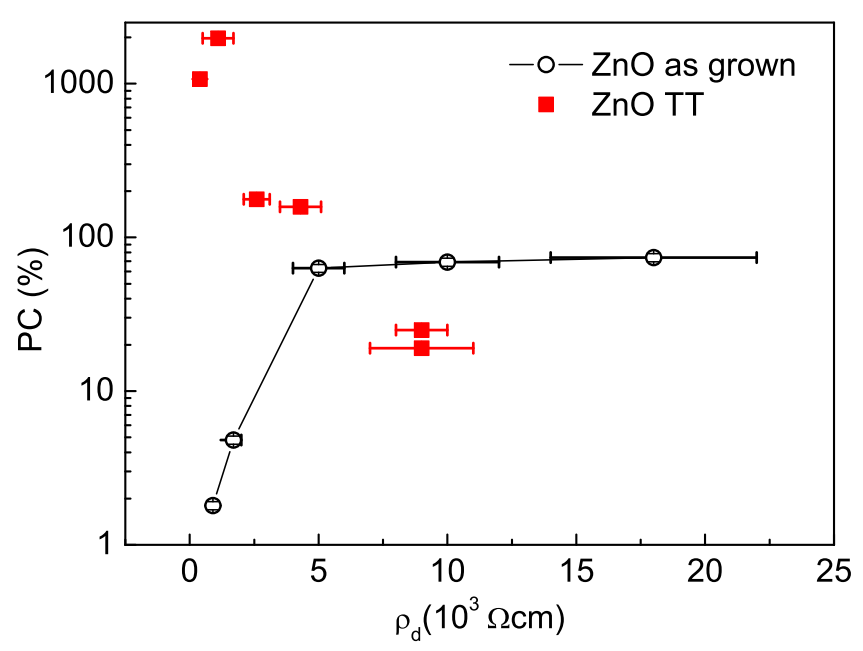

FIG. 6. Saturation photoconductivity at $1 \mathrm{~h}$ of illumination vs. dark resistivity of as-grown and TT microwires. The lines are guides to the eye.

Observing Fig. 6, we can infer that the MWs have grown with defects that affect simultaneously the $\rho_{d}$ and the PC response. As it is well established, the intrinsic defects give a type-n character to the pure $\mathrm{ZnO} .{ }^{26}$ Based on the discussion of the time constants of Fig. 5, the increase in the $\rho_{d}$ can be explained by an increase of the dominant intrinsic acceptor defects, i.e., zinc vacancies $\left(\mathrm{V}_{Z n}\right)^{24}$ that compensate the donor defects by capturing carriers. Then, the increase of concentration of $\mathrm{V}_{Z n}$ for higher $\rho_{d}$ correlates with the increase of PC, as it can be seen in Fig. 6 for as grown samples. In the case of TT samples, the behavior of PC vs. $\rho_{d}$ changes dramatically (see Fig. 6). The $V_{O}$ generated by the TT will have more concentration at surface or sub-surface sites, since the migration of $V_{O}$ is more favorable from inner sites to the surface. ${ }^{27}$ Furthermore, these $V_{O}$ are active traps for PC and then significantly enhance it for low $\rho_{d}$ compared with as-grown samples, as it is shown by the results of Fig. 6. Nevertheless, as $\rho_{d}$ increases, the PC drops almost two orders of magnitude in the measured range. The magnitude of the PC depends essentially on the photocurrent $\left(I_{p h}=I_{\text {light }}-I_{d}\right)$ through $\mathrm{PC}=I_{p h} / I_{d}$, where $I_{d}$ is the current in the dark. The concentration of photocarriers $\left(N_{p h}\right)$ can be evaluated as: $N_{p h}=(\eta \Phi / \mathrm{Al}) \tau$, where $\eta$ is the quantum efficiency, $\Phi$ is the photon absorption rate, A and 1 are the cross section and the length of the MW, respectively, and $\tau$ is the mean carrier lifetime. Then the photocurrent can be expressed as

$$
I_{p h}=\mathrm{e}(\eta \Phi / \mathrm{Al}) \tau \mathrm{vA}
$$

where $\mathrm{e}$ is the elementary charge, and $\mathrm{v}$ is the drift velocity. Using these results, the PC in steady state, in terms of $\rho_{d}$ and using $\mathrm{V}=\mu \mathrm{V} / \mathrm{l}$, where $\mathrm{V}$ is the applied voltage to the microwire, is

$$
\mathrm{PC}=\mathrm{e}(\eta \Phi / \mathrm{A} 1) \tau \mu \rho_{d}
$$

The mean carrier lifetime as a function of the photon absorption flux is ${ }^{3}$

$$
\tau=\tau_{2} /\left(1+\left(\Phi / \Phi_{0}\right)^{n}\right)
$$

where $\mathrm{n}$ is the phenomenological parameter, $\Phi_{0}$ is the photon absorption rate when trap saturation occurs, and $\tau_{2}$ is the slow time constant. Using Equations (1) and (2), we can obtain $\Phi_{0}$ for each sample. The results are shown in Fig. 7 , where a typical value of mobility for $\mathrm{ZnO}$ was used $\left(\mu \approx 10^{2} \mathrm{~cm}^{2} / \mathrm{V} \mathrm{s}^{28}\right)$ and taking $\mathrm{n}=0.7 .^{3}$ From Fig. 7 , we can see that $\Phi_{0}$ grows with $\rho_{d}$ for as grown samples, since $\Phi_{0}$ is proportional to the concentration of traps in the samples; this indicates that there are more imperfections in the samples with greater $\rho_{d}$. This conclusion supports our hypothesis that $V_{Z n}$ are responsible for the double effect of increasing PC and compensate donors as $\rho_{d}$ increases. Following the same reasoning with the TT samples, we can conclude from Fig. 7 that the concentration of traps decreases as $\rho_{d}$ increases. The same conditions of a given thermal treatment in vacuum will induce the same concentration of $V_{O}$ over different samples, so the different photoresponse comes from the interaction of them with the preexistent intrinsic defects. The previously reported existence of complex $\mathrm{V}_{Z n^{-}}-\mathrm{V}_{O}{ }^{29-31}$ is the more probable one (see Fig. 8). Since $\mathrm{V}_{Z n}$ are more mobile than $\mathrm{V}_{O}{ }^{32}$ they can migrate to the surface during the TT and reach the $\mathrm{V}_{O}$ to form the divacancy. ${ }^{33,34}$ This complex acts as a recombination center that fades out the photoresponse of the samples as its concentration increases. Then, for low $\rho_{d}$ the quantity of $V_{O}$, introduced by the TT, overpasses the number of preexistent $V_{Z n}$, relatively low concentration of complexes is formed, and the PC increases due to the new traps generated. As the concentration of $V_{Z n}$ increases, there is more probability to form complexes $\mathrm{V}_{Z n}-\mathrm{V}_{O}$ reducing the number of effective traps and then reducing the PC. This reduction is steeped due to the deactivation of the double role of the $\mathrm{V}_{Z n}$ : one as trap for photoconductivity and the other as compensating acceptor.

In Fig. 9, we show the PC vs. $\rho_{d}$ for as grown samples after hydrogenation by diffusion using the two processes described above: 30 bar at $300^{\circ} \mathrm{C}$ for $100 \mathrm{~min}$ (TTH1) and 40 bar at $450^{\circ} \mathrm{C}$ for $12 \mathrm{~h}(\mathrm{TTH} 2)$. The TTH1 has a similar effect over the samples as the TT in vacuum, a slight

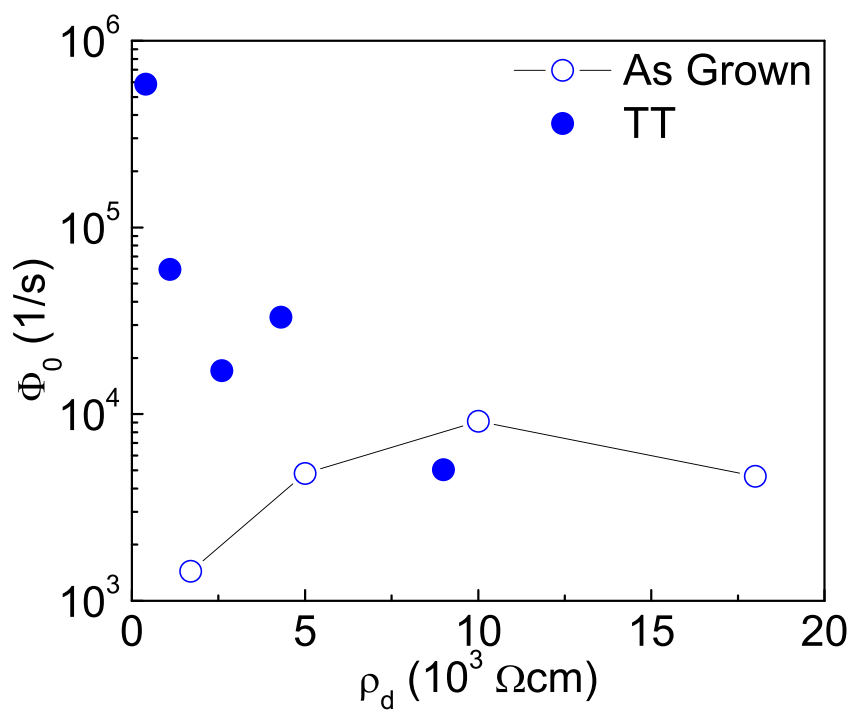

FIG. 7. Photon absorption rate for trap saturation as a function of dark resistivity for as grown and TT microwires. 


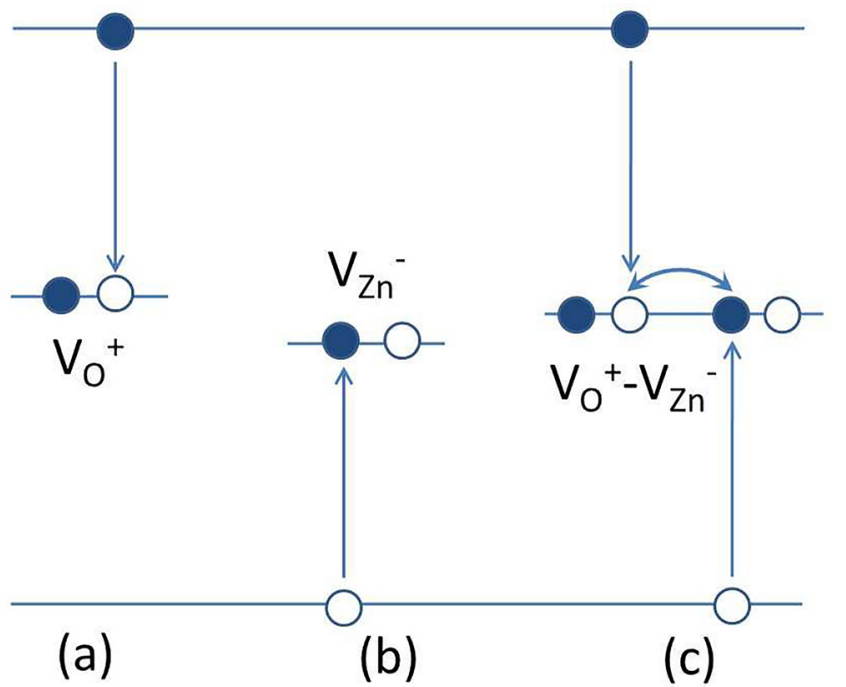

FIG. 8. (a) Single ionized oxygen vacancy acting as the trap of electrons. (b) Single ionized zinc vacancy acting as the trap of holes. (c) Neutral ZnO divacancy acting as the recombination center.

decrease on the $\rho_{d}$, and the same correlation between PC and $\rho_{d}$, but with lesser absolute values. As expected, the longer $\mathrm{TTH} 2$ process reduces the dark resistivity around two orders of magnitude below the other treatments. It was suggested that $\mathrm{H}$ is a robust source of n-type conductivity as substitutional at an $\mathrm{O}$ site $\left(H_{O}\right) .{ }^{35}$ The enhancement of the $\mathrm{PC}$ due to the hydrogenation is attributed to the bistability of the $H_{O}$ that can undergo a large lattice relaxation during illumination with UV, creating deep levels that trap electrons. ${ }^{36}$ Hence, the hydrogenation of our samples activates shallow preexistent $V_{O}$ producing the enhancement of the persistent photoconductivity. The decreasing trend of the PC against $\rho_{d}$ can be explained in the same way as TT samples; i.e., the $V_{Z n}$ deactivate the bistable $H_{O}$ traps forming a complex that acts as recombination centers. On the other side, the TTH2 samples have shown more dispersion in their PCs as a function on the $\rho_{d}$; nevertheless, the tendency is clear, they have a lesser PC than the other samples with thermal treatments. These samples have two orders of magnitude and less dark resistivity than the rest of the samples, which can be

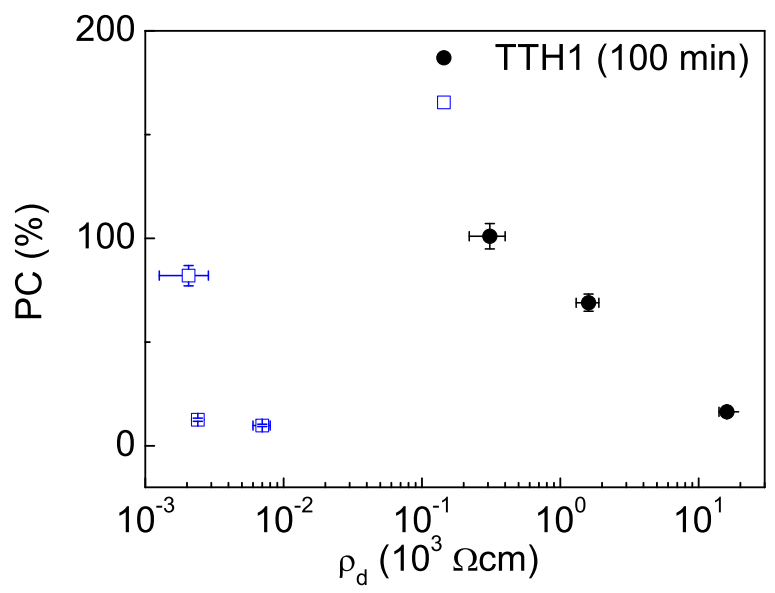

FIG. 9. Photoconductivity of hydrogenated MWs using two different processes: 30 bar at $300^{\circ} \mathrm{C}$ for $100 \mathrm{~min}$ or 40 bar at $450{ }^{\circ} \mathrm{C}$ for $12 \mathrm{~h}$. transduced in two orders of more carriers in the dark, and it is reasonable that the relative increment of the photocurrent will be lower, giving lesser PCs. From this point of view, it is interesting to note that a maximum photoresponse can be achieved by the interplay between concentration of carriers in the dark and intrinsic defects that are active traps for photoconductivity.

\section{CONCLUSIONS}

In summary, we have studied the photoconductivity as a function of the resistivity in dark of single $\mathrm{ZnO}$ microwires, grown by carbothermal reduction. The photoconductivity of as grown samples increases with dark resistivity due to the increase of zinc vacancies that compensate donors and act as traps for photoconductivity. In thermal treated samples in vacuum, the correlation between photoconductivity and dark resistivity is inverted due to the formation of divacancies of $\mathrm{ZnO}$ in the surface of the microwires that act as recombination centers that fade out steeply the photoconductivity as the dark resistivity increases. To conclude, we have shown that the $\mathrm{ZnO}$ divacancies can play a relevant role in the photoconductor behavior in microwires with thermal treatments in reducing atmospheres.

\section{ACKNOWLEDGMENTS}

This work was supported by PIUNT E530 CX (SCAITUNT), FSTics 002/2010 (FONARSEC), and SNMAG and LNLS (Campinas, Brazil) facilities. We thank C. E. Rodriguez Torres for her assistance on XAS measurements.

${ }^{1}$ T. Zhai, X. Fang, M. Liao, X. Xu, H. Zeng, B. Yoshio, and D. Golberg, Sensors 9, 6504 (2009).

${ }^{2}$ K. Liu, M. Sakurai, and M. Aono, Sensors 10, 8604 (2010).

${ }^{3}$ C. Soci, A. Zhang, B. Xiang, S. Dayeh, D. Aplin, J. Park, X. Bao, Y. Lo, and D. Wang, Nano Lett. 7, 1003 (2007).

${ }^{4}$ L. W. Ji, S. M. Peng, Y. K. Su, S. J. Young, C. Z. Wu, and W. B. Cheng, Appl. Phys. Lett. 94, 203106 (2009).

${ }^{5}$ R. S. Aga, D. Jowhar, A. Ueda, Z. Pan, W. E. Collins, R. Mu, K. D. Singer, and J. Shen, Appl. Phys. Lett. 91, 232108 (2007).

${ }^{6}$ M.-W. Chen, C.-Y. Chen, D.-H. Lien, Y. Ding, and J.-H. He, Opt. Express 18, 14836 (2010).

${ }^{7}$ S. Dhara and P. K. Giri, J. Appl. Phys. 110, 124317 (2011).

${ }^{8}$ H. Li, X. Zhang, N. Liu, L. Ding, J. Tao, S. Wang, J. Su, L. Li, and Y. Gao, Opt. Express 23, 21204 (2015).

${ }^{9}$ A. Bera, T. Ghosh, and D. Basak, ACS Appl. Mater. Interfaces 2, 2898 (2010).

${ }^{10}$ Y. N. Hou, Z. X. Mei, H. L. Liang, D. Q. Ye, C. Z. Gu, and X. L. Du, Appl. Phys. Lett. 102, 153510 (2013).

${ }^{11}$ S. Sarkar and D. Basak, Appl. Phys. Lett. 103, 041112 (2013).

${ }^{12}$ L. Shi, F. Wang, B. Li, X. Chen, B. Yao, D. Zhao, and D. Shen, J. Mater. Chem. C 2, 5005 (2014).

${ }^{13}$ O. Lupan, V. Cretu, V. Postica, M. Ahmadi, B. R. Cuenya, L. Chow, I. Tiginyanu, B. Viana, T. Pauport, and R. Adelung, Sens. Actuators, B 223, 893 (2016).

${ }^{14}$ K. C. Hui, H. C. Ong, P. F. Lee, and J. Y. Dai, Appl. Phys. Lett. 86, 152116 (2005).

${ }^{15}$ A. Bera and D. Basak, ACS Appl. Mater. Interfaces 2, 408 (2010).

${ }^{16}$ C. Park, J. Lee, H.-M. So, and W. S. Chang, J. Mater. Chem. C 3, 2737 (2015).

${ }^{17}$ P. Ivanoff Reyes, C.-J. Ku, Z. Duan, Y. Xu, E. Garfunkel, and Y. Lu, Appl. Phys. Lett. 101, 031118 (2012).

${ }^{18}$ S. Mondal, R. Ram Ghimire, and A. K. Raychaudhuri, Appl. Phys. Lett. 103, 231105 (2013).

${ }^{19}$ B. Q. Cao, M. Lorenz, M. Brandt, H. von Wenckstern, J. Lenzner, G. Biehne, and M. Grundmann, Physica Status Solidi (RRL) 2, 37 (2008). 
${ }^{20}$ N. Ashkenov, B. N. Mbenkum, C. Bundesmann, V. Riede, M. Lorenz, D. Spemann, E. M. Kaidashev, A. Kasic, M. Schubert, M. Grundmann, G. Wagner, H. Neumann, V. Darakchieva, H. Arwin, and B. Monemar, J. Appl. Phys. 93, 126 (2003).

${ }^{21}$ J. C. Moore and C. V. Thompson, Sensors 13, 9921 (2013).

${ }^{22}$ Q. H. Li, T. Gao, Y. G. Wang, and T. H. Wang, Appl. Phys. Lett. 86, 123117 (2005).

${ }^{23}$ S. A. Studenikin, N. Golego, and M. Cocivera, J. Appl. Phys. 83, 2104 (1998).

${ }^{24}$ F. Tuomisto, V. Ranki, K. Saarinen, and D. C. Look, Phys. Rev. Lett. 91, 205502 (2003).

${ }^{25}$ M. Villafuerte, J. M. Ferreyra, C. Zapata, J. Barzola-Quiquia, F. Iikawa, P. Esquinazi, S. P. Heluani, M. M. de Lima, Jr., and A. Cantarero, J. Appl. Phys. 115, 133101 (2014).

${ }^{26}$ S. B. Zhang, S.-H. Wei, and A. Zunger, Phys. Rev. B 63, 075205 (2001).

${ }^{27}$ B. Deng, A. Luisa da Rosa, T. Frauenheim, J. P. Xiao, X. Q. Shi, R. Q. Zhang, and M. A. Van Hove, Nanoscale 6, 11882 (2014).
${ }^{28} \mathrm{H}$. Morkoc and U. Ozgur, Zinc Oxide, Fundamentals, Materials and Device Technology, 1st ed. (Wiley-VCH Verlag GmbH \& Co. KGaA, Federal Republic of Germany, 2009).

${ }^{29}$ S. A. Studenikin and M. Cocivera, J. Appl. Phys. 91, 5060 (2002).

${ }^{30}$ R. Vidya, P. Ravindran, H. Fjellvåg, B. G. Svensson, E. Monakhov, M. Ganchenkova, and R. M. Nieminen, Phys. Rev. B 83, 045206 (2011).

${ }^{31}$ Y.-J. Zeng, K. Schouteden, M. N. Amini, S.-C. Ruan, Y.-F. Lu, Z.-Z. Ye, B. Partoens, D. Lamoen, and C. V. Haesendonck, ACS Appl. Mater. Interfaces 7, 10617 (2015).

${ }^{32}$ P. Erhart and K. Albe, Appl. Phys. Lett. 88, 201918 (2006).

${ }^{33}$ E.-H. Shin and H. Kim, J. Korean Phys. Soc. 66, 625 (2015).

${ }^{34}$ M. S. Holston, E. M. Golden, B. E. Kananen, J. W. McClory, N. C. Giles, and L. E. Halliburton, J. Appl. Phys. 119, 145701 (2016).

${ }^{35}$ A. Janotti and C. Van de Walle, Nat. Mater. 6, 44 (2007).

${ }^{36}$ H.-H. Nahm, C. H. Park, and Y.-S. Kim, Sci. Rep. 4, 4124 (2014). 